\title{
Ethnic variation in fluorescein angiography induced nausea and vomiting
}

\begin{abstract}
Purpose A prospective survey was undertaken to investigate ethnic variations in the frequency of nausea and vomiting after fundus fluorescein angiography (FFA). Method Between May and September 1998, 197 adult patients were recruited to the study. A questionnaire containing closed-ended questions was completed by nurses after each FFA and a questionnaire was given to patients to complete 5 hours after the procedure at home. Patients' anxiety level was measured before FFA using a 5-item ordinal response scale.
\end{abstract}

Results Results indicate that patients from black, Asian, Chino-Asian and mixed ethnic origins are significantly more likely to vomit and feel nauseous immediately after the administration of fluorescein dye. Patients with a history of nausea after FFA are significantly more likely to feel nauseous again after repeat FFA.

Conclusion Ethnic origin and a previous history of nausea and vomiting appear to be important factors in FFA-induced nausea and vomiting. The results of this study have led the investigators to develop a protocol for the prophylactic treatment of nausea and vomiting following FFA.

Key words Ethnic, Fluorescein angiography, Nausea, Ophthalmic, Ophthalmology, Vomiting

Fundus fluorescein angiography (FFA is a diagnostic study of the circulation of the dye through the retina of the eye. It is a widely used investigative and monitoring procedure for patients with diabetic retinopathy and other retinal diseases. It was observed, in the clinical setting, that black or Asian patients appeared to be nauseous and vomit more frequently than their white counterparts after FFA. This led to an investigation of the frequency of nausea and vomiting after administration of fluorescein dye.
The term 'ethnic group' refers to a 'group having a common national or cultural tradition; denoting origin by birth or descent rather than nationality'. ${ }^{1}$

\section{Literature review}

There has been no research investigating ethnic origin and nausea and vomiting after FFA; however, the effects of other factors have been studied. These include the concentration of fluorescein injected, velocity of injection, use of pre-medication and history of nausea and vomiting.

Adverse reactions after intravenous fluorescein are usually mild, consisting of immediate nausea and vomiting. According to Kwiterovich et al. ${ }^{2}$ the reported frequency of all adverse reactions varies from $0.6 \%$ to $16.1 \%^{3-5}$ and early reports on nausea vary from $2.2 \%{ }^{5}$ to $15 \%{ }^{6}$ The frequency of vomiting varies from $1 \%{ }^{7}$ to $3.3 \%{ }^{4}$

One study found that in patients who had multiple angiograms, $77 \%$ of the nausea reaction occurred after the patient's first injection, with no further reaction following subsequent injections. ${ }^{6}$

Kwiterovich et al. ${ }^{2}$ also highlight that previous research has shown no significant difference in the frequency of reaction using $5 \%$, $10 \%$ or $25 \%$ concentration of fluorescein. ${ }^{5-8}$ However, Yanuzzi et al. report that there may have been differences in the rate of nausea between different concentrations, i.e. $5 \%$ and $10 \%$, of fluorescein. ${ }^{9}$ No difference in frequency of adverse reactions was found between two rates (fast and slow) of injection speed, when the slow rate is defined as an injection lasting longer than $6 \mathrm{s.}^{2}$

Kwiterovich et al. ${ }^{2}$ investigated whether the frequency of adverse reaction to FFA was related to a history of previous angiography with or without a history of adverse reaction. This involved 2789 consecutive angiography procedures in 2025 patients. They report that in those patients who had no history of adverse reactions the incidence of adverse reactions was $1.8 \%$, whereas those patients who had a history
R. McLauchlan Retinal Medical Unit Manchester Royal Eye Hospital

Manchester, UK

H. Waterman

C. Dodd

Manchester Royal Eye

Hospital

Manchester, UK

H. Waterman

C. Waterman

School of Nursing

Midwifery and Health

Visiting

University of Manchester

Manchester, UK

V. Hillier

Imaging Science and Biomedical Engineering University of Manchester Manchester, UK

Heather Waterman, PhD, BSc, RGN, OND School of Nursing, Midwifery and Health Visiting

University of Manchester Oxford Road

Manchester M13 9PL, UK

Tel: +44 (0)161 2756960

Fax: +44 (0)161 2755346 e-mail:

heather.waterman@man.ac.uk

Received: 9 March 2000

Accepted in revised form:

5 September 2000 
of adverse reactions $48.6 \%$ experienced adverse reactions again. The results of this study demonstrate the importance of a patient's angiography history, but do not help health professionals to care for those who attend for the first time.

However, there are a number of other factors such as age, sex, psychological or medical state of patients which can also affect reaction to dye. Male patients were found to react adversely more frequently than females to the use of intravenous fluorescein, ${ }^{10}$ whilst a higher complication rate in individuals 10 years younger than the average patient age was found in another study. ${ }^{11}$ Chazan et al..$^{6}$ noted a higher incidence of nausea when patients were asked specifically whether they experienced nausea.

This review identifies a gap in the literature in that it appears that no previous study has addressed the ethnic variation in reaction to fluorescein dye. The objectives of the survey were:

1. to find out the frequency of nausea and vomiting following FFA;

2. to find out whether certain ethnic groups were more prone to nausea and vomiting following FFA;

3. to explore the effect of other variables which may be related to nausea and vomiting, i.e. age, sex, anxiety and perceived discomfort from the camera light.

\section{Patients and methods}

One hundred and ninety-seven patients, who were attending either for the first time or for repeat fluorescein angiography, were recruited consecutively to the study. The target population was all male and female adults undergoing FFA between May and October 1998. After the preliminary analysis, it was found that the proportion of patients of black, Asian, Chino-Asian or mixed ethnic origin was too small for the application of statistical tests. Thereafter, on the advice of an experienced medical statistician, only those patients of black, Asian, ChinoAsian or mixed ethnic origin were approached to take part in order to obtain a larger sub-sample.

The survey was conducted in the Retinal Unit at Manchester Royal Eye Hospital, where FFA is undertaken by experienced ophthalmic nurses.

Questionnaires were administered and completed by appropriately trained ophthalmic nurses at each FFA examination. Another questionnaire was given to patients to complete 5 hours after the procedure in order to identify any problems that may have occurred during the journey home or at home. Stamped addressed envelopes were provided to patients to encourage return of questionnaires.

Given the lack of previous studies in this particular area, the questionnaire was developed for the purpose of the study. Item topics were generated from the literature and from suggestions from nurses, doctors and anaesthetists. The questionnaire was piloted, reviewed and revised as necessary. The final questionnaire that nurses completed consisted of 12 closed-ended items, and the questionnaire for patients contained 7 items.
Information on the following variables was collected: diagnosis, new or previous patient, sex, age, date of birth, nausea, vomiting, ethnic group (grouped according to NHS categories), medication including anti-emetics, past history of nausea after FFA, motion sickness, anxiety, discomfort and light from camera, and nausea and vomiting at home.

Patients' anxiety levels were measured in order to investigate a possible relationship between pre-FFA anxiety and post-FFA nausea. Ordinal response scales were employed to gain answers to the following queries on anxiety: (1) Were you worried about the procedure? (2) Was the procedure on your mind continually? The two extremes are anchored by responses such as 'not at all', 'slightly', 'moderately', 'very' and 'extremely'. These two questions were modified from the Amsterdam Preoperative Anxiety Scale. ${ }^{12}$ The Amsterdam Scale has four questions, two of which were not relevant for our purposes. No other small and 'easy to use' scale could be located on the subject of pre-procedural anxiety. Most patients appeared to understand the questions and ambiguities were clarified at the time of the procedure. Nurses were trained in how to complete the questionnaire.

The study was discussed with the chairman of the local research ethics committee, who felt ethics committee approval was not necessary. The approval of the Senior Nurse and all consultants of patients involved were obtained. Each patient was informed of the study and consented to participate.

All data obtained were quantified and analysed using the Statistical Package for Social Sciences (SPSS). The chisquare test, Fisher's Exact test and Mann-Whitney $U$-test were applied where appropriate.

\section{Results}

Diagnosis

Table 1 shows the distribution of eye diseases among the sample. Patients with diabetic maculopathy form the largest group.

\section{New patients}

Just over two-thirds of patients $(127,67.9 \%)$ were new to this procedure (10 missing data).

Table 1. Diagnoses

\begin{tabular}{lc}
\hline Diagnosis & Frequency \\
\hline Diabetic maculopathy & 69 \\
Macular changes & 55 \\
SRNVM & 27 \\
Macular oedema & 19 \\
CRVO & 14 \\
ARMD & 11 \\
Choroidal melanoma & 1 \\
Missing & 1 \\
Total & 197 \\
\hline
\end{tabular}

SRNVM, subretinal neovascular membrane; CRVO, central retinal vein occlusion; $A R M D$, age-related macular degeneration 
Table 2. Frequency of ethnic groups

\begin{tabular}{lc}
\hline Ethnic group & Frequency \\
\hline White & 157 \\
Black & 5 \\
Asian & 25 \\
Chino-Asian & 1 \\
Others & 5 \\
Mixed race & 3 \\
Missing & 1 \\
Total & 197 \\
\hline
\end{tabular}

Age

The age range was from 20 to 89 years with mean age of 60 years (SD 15.3; 2 missing data).

\section{Nausea and vomiting following FFA}

Twenty (10.6\%) patients reported feeling nauseous (9 missing data) and 8 (4.2\%) vomited after FFA (7 missing data). Vomiting occurred immediately after FFA (range 5-210 s); 2 subjects vomited five and three times respectively. Using the chi-square test, no significant difference was found in the proportion of males and females reporting nausea $(p=0.979)$.

\section{Ethnic group}

The proportions of patients from different ethnic groups are found in Table 2; white patients formed the largest group (157, 80.1\%; 1 missing data). Table 3 contains data on the frequency of nausea and vomiting in each ethnic group. In order to perform statistical tests, the ethnic groups were categorised as white or black (black, Asian, Chino-Asian, mixed race). Using Fisher's Exact test, the black group were significantly more likely to feel nauseous $(p=0.00385)$ and vomit $(p=0.00908)$ than the white group. Within the white and black groups, using the chi-square test there were no differences in the proportions of males and females $(p=1.0)$.

\section{Medication}

It was identified that $3(1.5 \%)$ patients had taken an oral anti-emetic prescribed by their general practitioner prior to the procedure ( 0 missing data). Of these 3 , only 1 patient took the anti-emetic as a precaution against

Table 3. Frequency of nausea and vomiting in each ethnic group

\begin{tabular}{|c|c|c|}
\hline Ethnic group & Nausea & Vomiting \\
\hline White & $10(140)$ & $3(148)$ \\
\hline Black & 1 (4) & $1 \quad(4)$ \\
\hline Asian & $6 \quad(17)$ & $4 \quad(20)$ \\
\hline Chino-Asian & $0 \quad(1)$ & $0 \quad(1)$ \\
\hline Mixed race & $0 \quad(3)$ & $0 \quad(3)$ \\
\hline Others & $2 \quad(3)$ & $0 \quad(5)$ \\
\hline Total & $19(168)$ & $8(181)$ \\
\hline
\end{tabular}

Frequency of patients who were not nauseous and who did not vomit are in parentheses. nausea and vomiting. The other 2 patients were taking the anti-emetic for other reasons. None of these patients was nauseous or vomited.

\section{Previous history of nausea and vomiting following FFA}

Eleven (10.6\%) patients had a history of nausea and 2 $(1.9 \%)$ patients had a history of vomiting following FFA (3 missing data). Seven $(63.6 \%$ ) patients who had a past history of nausea were also nauseous again. Of those patients with no history of nausea, $82(92.1 \%)$ went on to report no nausea again. Using Fisher's Exact test, patients with a history of nausea following FFA were significantly more likely to be nauseous $(p=0.001)$

\section{Motion sickness}

A history of motion sickness was noted in 25 (13.4\%) patients ( 5 missing data). Using the chi-square test, there were no significant differences between those who reported motion sickness and those who did not in the proportion of either those who were nauseous or those who vomited ( $p=0.478, p=0.596$ respectively).

\section{Anxiety}

There were no significant differences in the two anxiety scores before FFA between those who vomited and those who did not $(Z=-1.748, p=0.0804 ; Z=-1.802$, $p=0.0715$ ) using the Mann-Whitney $U$-test. Surprisingly non-vomiters reported slightly higher anxiety scores in all ethnic groups. There was also no significant relationship between nausea after FFA and anxiety (question $1: Z=-0.423, p=0.673$; question $2: Z=-0.027$, $p=0.979)$.

\section{Light from camera}

A question was asked of the subjects regarding the light from the camera: $28(14.2 \%)$ said that they felt uncomfortable (44 missing data). There was no significant difference between those who felt nauseous and those who did not in the proportion finding the light uncomfortable.

\section{At home}

The results from the subjects who returned the questionnaire from home $(153,78 \%)$ were interesting. No patients vomited on their journey home or at home; however, $18(11.8 \%)$ patients felt nauseous. Of the patients who were nauseous at home, 13 were classed as 'white', 4 were categorised as 'Asian' and 1 was deemed 'other'. Fourteen patients who did not report nausea immediately after the FFA injection felt nauseous at home (1 missing data). 


\section{Discussion}

Although the sample size precludes a definitive conclusion, the results strongly suggest that black, Asian, Chino-Asian and mixed race patients are significantly more likely to feel nauseous and vomit after administration of fluorescein. The numbers were too small to isolate different ethnic groups. Future research might investigate the effect of differing ethnic groups. No information could be found which offered an explanation for the ethnic variation. It is assumed that there is some physiological difference in nausea and vomiting mechanisms but further research is required to explore this thesis further.

This study supports the findings of Kwiterovich et al. ${ }^{2}$ that patients with a history of nausea were significantly more likely to be nauseous and vomit after FFA. Our results also suggest, however, that another factor, i.e. ethnic group, may play a part in determining the likelihood of nausea and vomiting and also helps to explain the variations in past reported incidences.

In the light of our results, previous research and on the recommendation of hospital pharmacists and a consultant anaesthetist, Buccastem (buccal prochlorperazine) $6 \mathrm{mg}$ is now being tested with a view to reducing the rate of nausea and vomiting following FFA. It is being given to patients of one consultant who either have a history of nausea and vomiting, or are new patients of black, Asian, Chino-Asian or mixed race. Buccastem has the advantage of being relatively quick in its action without the need for an intramuscular or intravenous injection, is relatively easy to administer in a busy clinic, is effective in the vast majority of patients and is widely used throughout Central Manchester Health Care Trust to treat post-operative nausea.

It is interesting that our results do not support a relationship between high levels of anxiety and nausea; indeed it appears that lower levels of anxiety were associated with nausea. This appears to be contrary to current thinking and we cannot offer any explanations for this phenomenon.

The overall incidence of nausea (10.2\%) in our study was within previously reported ranges of $2.9 \%{ }^{3}$ to $15 \%{ }^{7}$ The overall incidence of vomiting $(4.2 \%)$ was beyond previous ranges of $1 \%{ }^{8}$ to $3.3 \% .^{5}$ The higher reported levels in our study may be due to the higher proportion of sampled black, Asian, Chino-Asian and mixed race patients.

In summary, this survey has significant findings which have a number of clinical and theoretical implications. The results strongly suggest that ethnic origin is a factor in post-FFA nausea and vomiting. The results also support previous research that had indicated patients with a history of FFA-induced nausea are significantly more likely to be nauseous on repeat FFA. The survey also appeared to show that confounding variables such as age, sex, anxiety level and perceived discomfort from the camera light had little impact on nausea and vomiting. These results present some challenges for clinical practice. The implications for health professionals must be considered and future research could explore the reasons why patients from black, Asian, Chino-Asian and mixed ethnic origins appear to be more susceptible to nausea and vomiting.

We would like to thank all staff and patients who helped with this survey.

\section{References}

1. Anon. Skin deep. Education Guardian 1992;19 May:1-3.

2. Kwiterovich $K$, Maguire MG, Murphy RP, Schachat AP, Bressler NM, Bressler SB, Fine SL. Frequency of adverse reactions after fluorescein angiography. Ophthalmology 1991;97:1139-42.

3. Stein MR, Parker CW. Reactions following intravenous fluorescein. Am J Ophthalmol 1971;72:861-8.

4. Yannuzzi LA, Justice J, Baldwin HA. Effective differences in the formulation of intravenous fluorescein and related side effects. Am J Ophthalmol 1974;78:217-21.

5. Butner RW, McPherson AR. Adverse reactions in intravenous fluorescein angiography. Ann Ophthalmol 1983;15:1084-6.

6. Chazan BI, Balodimos MC, Koncz L. Untoward effects of fluorescein retinal angiography in diabetic patients. Ann Ophthalmol 1971;3:42-9.

7. Marcus DF, Bovino JA, Williams D. Adverse reactions during fluorescein angiography [letter]. Arch Ophthalmol 1984;102:825.

8. Justice J, Paton D, Beyer CR, Seddon GG. Clinical comparison of $10 \%$ and $25 \%$ intravenous sodium fluorescein solutions. Arch Ophthalmol 1977;95:2015-6.

9. Yannuzzi LA, Rohrer KT, Tindel LJ, Sobel RS, Costanza MA, Shields W, Zang E. Fluorescein angiography complication survey. Ophthalmology 1986;93:611-7.

10. Greene GS, Bell LW, Hitching RA, Spaeth GL. Adverse reaction to intravenous fluorescein: evidence for sex difference. Ann Ophthalmol 1976;8:533-6.

11. Pacurariu RI. Low incidence of side effects following intravenous fluorescein angiography. Ann Ophthalmol 1982;14:32-6.

12. Moerman N, Frits SAM, Van Dam Muller MJ, Oosting H. Amsterdam Pre-operative Anxiety and Information Scale (APAIS). Anaesth Analg 1996;82:445-51. 